\title{
Development of Lattice QCD Tool Kit on Cell Broadband Engine Processor
}

\section{Shinji Motoki*}

Graduate School of Bio-Sphere Science, Hiroshima University, Higashi-Hiroshima, Hiroshima, 739-8521, Japan

E-mail: motoki-shinji@hiroshima-u.ac.jp

\section{Yoshiyuki Nakagawa}

Research Institute for Information Science and Education, Hiroshima University,

Higashi-Hiroshima, Hiroshima, 739-8521, Japan

E-mail: nkgw@rcnp.osaka-u.ac.jp

\section{Keitaro Nagata}

Research Institute for Information Science and Education, Hiroshima University, Higashi-Hiroshima, Hiroshima, 739-8521, Japan

E-mail: nagata@rcnp.osaka-u.ac.jp

\section{Koichi Hashimoto}

Fixstars Corporation, Nisshin Bldg. 3F, 1-8-27, Kounan, Minato-ku, Tokyo 108-0075, Japan

E-mail: hashimotodixstars.com

\section{Kiyoshi Mizumaru}

Fixstars Corporation, Nisshin Bldg. 3F, 1-8-27, Kounan, Minato-ku, Tokyo 108-0075, Japan

E-mail: marulefixstars.com

\section{Atsushi Nakamura}

Research Institute for Information Science and Education, Hiroshima University,

Higashi-Hiroshima, Hiroshima, 739-8521, Japan

E-mail: nakamura@riise.hiroshima-u.ac.jp

We report an implementation of a code for SU(3) matrix multiplication on Cell/B.E., which is a part of our project, Lattice Tool Kit on Cell/B.E.. On QS20, the speed of the matrix multiplication on SPE in single precision is 227GFLOPS and it becomes 20GFLOPS together with data transfer from main memory by DNA transfer, which is $4.6 \%$ of the hardware peak speed (460GFLOPS), and is $7.4 \%$ of the theoretical peak speed of this calculation (268.77GFLOPS). We briefly describe our tuning procedure.

The XXVII International Symposium on Lattice Field Theory - LAT2009

July 26-31 2009

Peking University, Beijing, China

\footnotetext{
* Speaker.
} 


\section{Introduction}

This paper has three objectives;

1. We report a development of a SU(3) matrix multiplication code on the Cell/B.E.. This calculation is, needless to say, an essential numerical part of any quench QCD calculation, and we expect that Cell/B.E. can be a good cost effective environment for a quench QCD study such as the confinement, transport coefficients etc..

2. We report several techniques to improve our Cell program performance, which we found during developing our code. They are general and can be used to develop other scientific high-performance code.

3. The experience reported here is a starting point to develop a whole QCD simulation system on Cell/B.E., including the quark fermion matrix solver, which is now the most time consuming part of lattice QCD. Even a single Cell machine makes it possible for a researcher or a small research group to calculate quark dynamics. And large scale Cell machine may work as a most powerful QCD machine[4]

Lattice QCD society has been always looking for a powerful computer for more than thirty years to realize their dream, i.e., to simulate QCD on a computer, to produce reliable data, and to understand non-perturbative nature of QCD including the confinement, hadron interactions, and quank-gluon plasma. Machines for this aim were VAX11, vector machines, parallel computers, clusters, GRID and so on.

The Cell/B.E. has attracted much interest in scientific and engineering fields as a high performance machine $[1,2,3,4]$. It has very high potential power, and is a much more reliable scientific computing system comparing current GP/GPU.

In order to extract its potential power, programing on Cell/B.E. demands several cares, which were not required on the traditional computers. This is because Cell/B.E. is a machine of new architecture and has the following features:

Multi-core computer: A Cell/B.E. consists of eight operation system processor cores called Synergistic Processor Element(SPE) and one system controll processor core called PowerPC Processor Element (PPE).

SIMD operation: The SPE is specialized to calculations in the use and has the new architecture with the ability of the SIMD operation.

Small local memory LS: SPE has a local store (LS) of 256 KBytes which worked as an inside memory of SPE.

EIB connection and DMA: PPE and all SPE are connected by a high-speed bus called Element Interconnect Bus (EIB). The EIB is also connected to main memory and to an input and output device. Each processor core performs data access via the EIB.

SPE uses Direct Memory Access (DMA) transfer for data transmission. The DMA is used to forward data directly between memory and memory (or, memory and input/ output device). 
Although the EIB connection provides fast transfer of the data, the transfer time should be hidden to get satisfactory performance.

Large number of registers: Each SPE has 128 general registers. It is important to use this advantage to write an efficient code.

In this report, we consider $S U(3)$ matrix multiplication, $c^{(i)}=a^{(i)} \times b^{(i)}$ for $i=0, N-1$. We show a result for $N=573,440$.

The hardware peak speed of QS20, which has 2PPE and 16SPE, is 460GFlops, while theoretical peak speed of this calculation on Cell/B.E. is 268.769518291 GFLOPS.

\section{Tuning Procedure}

\subsection{Optimized Data Handling}

In order to extract SPE's calculational power, the full use of its Single Instruction Multiple Data(SIMD) function is essential. We must provide our input matrix data in a form which fits the SIMD operation. The SPE can handle several data at once by one instruction with vector data type. The data treated in the Cell/B.E. are 16 bytes fixation, and it can handle four data at once by a single instruction in the single precision floating point arithmetic.

When executing the DMA data transformation between the LS and main memory, a 128 byte aligned data structure is most efficient. The maximum amount of data at one transfer is $16 \mathrm{KByte}$, and it is desirable for data to fit this restriction.

We pack the matrices, $a$ and $b$, of 16KByte in a structure. In order to fit the algorithm done on SPE, we separate the real and imaginary parts of a complex matrix, and pack 112 matrices.

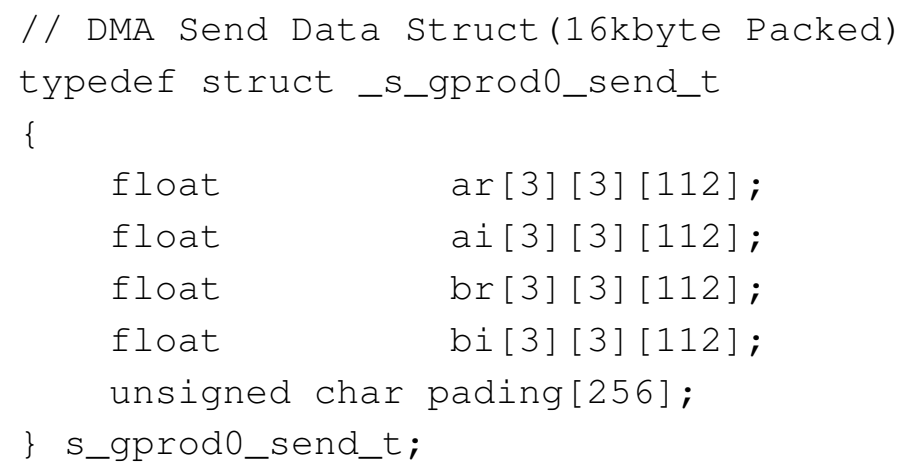

\subsection{Effect of SIMDizaiton}

The SIMD operation is the operation technique that can process plural data by one instruction.[7] When we use only PPE, the above calculation takes 365.080039 (msec), while it is 40.55844 (msec) when we use SIMD (1SPE). Furthermore, when we employ 16SPE it takes 10.13458 (msec).

\subsection{Multi Buffering}

In the parallel calculation, data transmission often becomes the bottleneck, except trivially parallel applications.[5, 6] Therefore we must conceal the time for DMA transfer between the main memory and $\mathrm{LS}^{1}$.

\footnotetext{
${ }^{1}$ The calculation described here does not require the data transfer among SPE's.
} 
For this purpose, we adopt a technique called double- or multi- buffering. First let us consider a double buffering case for transforming the matrices $a$ and $b$ from the main memory to each LS of sixteen SPE's. We prepares two sets of buffers:

- We start to transfer the input data in the first buffer by DMA to SPE's LS.

- When the data arrive, the SPE executes the matrix multiplication.

- Without interruption, Memory Flow Controller(MFC) continues to send the next data in the second buffer. This transfer time overlaps with the matrix-multiplication calculation time. Thus part of the transfer time is "hidden".

- MFC continues to send the next data in the first buffer.
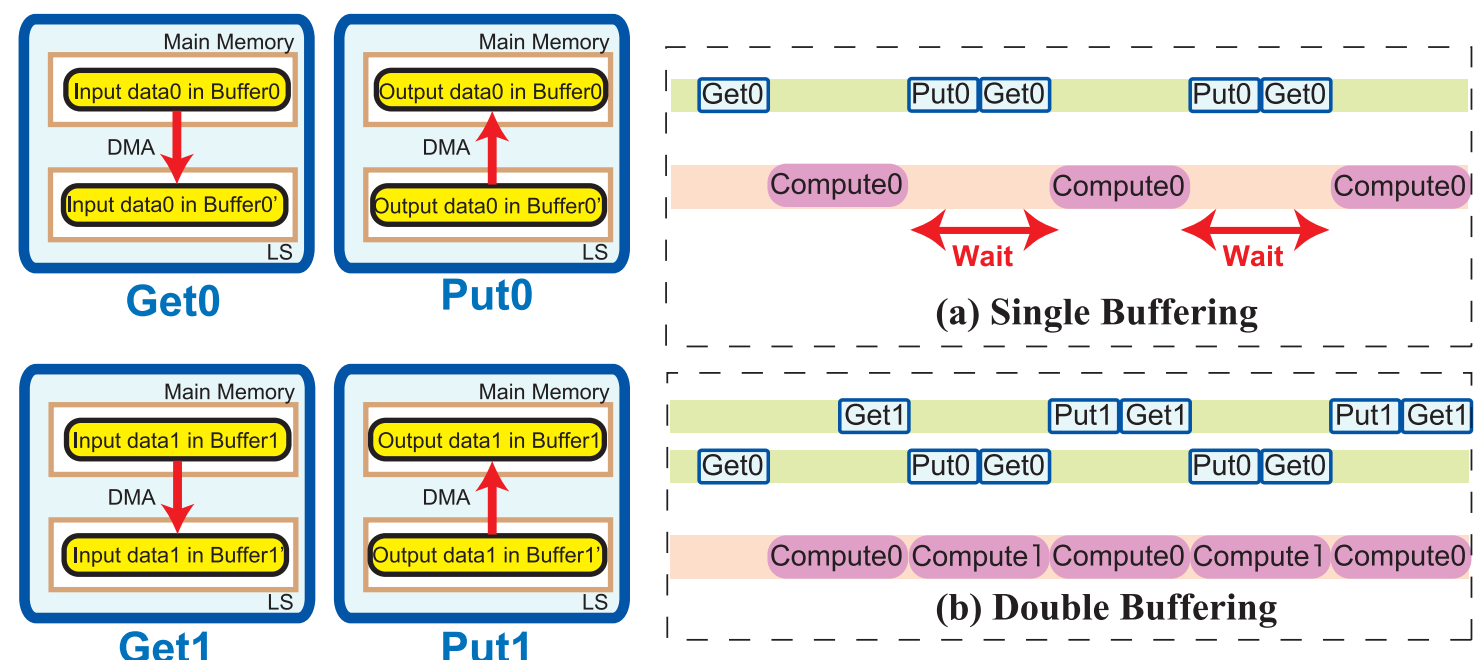

(a) Single Buffering

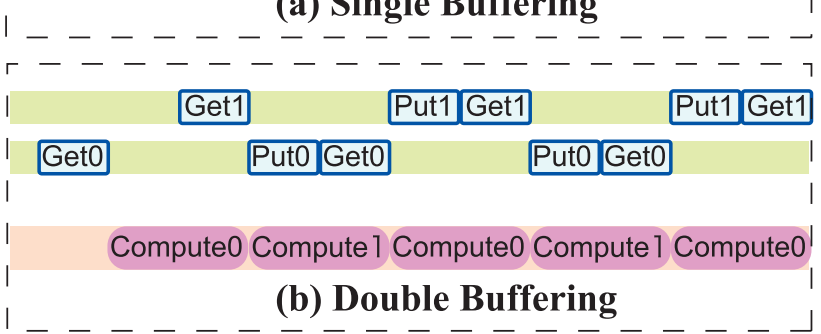

Figure 1: Single and Double Buffering

In Fig.1, we show a schematical diagram of (a)Single and (b)Double Buffering. When we use Single Buffering (16SPEs), it takes 10.1385 (msec), while it takes 9.1241 (msec) when we employ Double Buffering(16SPEs). SIMD improves the efficiency three to four times. On the other hand, the effect of the double buffering is around $10 \%$.

\subsection{Loop Unrolling and Software Pipelining}

The SPE has 128 general registers of 128 bit. Thanks to the many registers, loop unrolling results in a high-performance code (see Fig. 2). In addition, the SPE has the pipeline of two asymmetries, and two instructions can be executed at once. The speedup can be achieved by considering it.

Present compilers are not strong enough to find the most optimal level of the loop unrolling for a CPU of such many registers. Thus, we must optimize a code by hand. We develop a loop by 

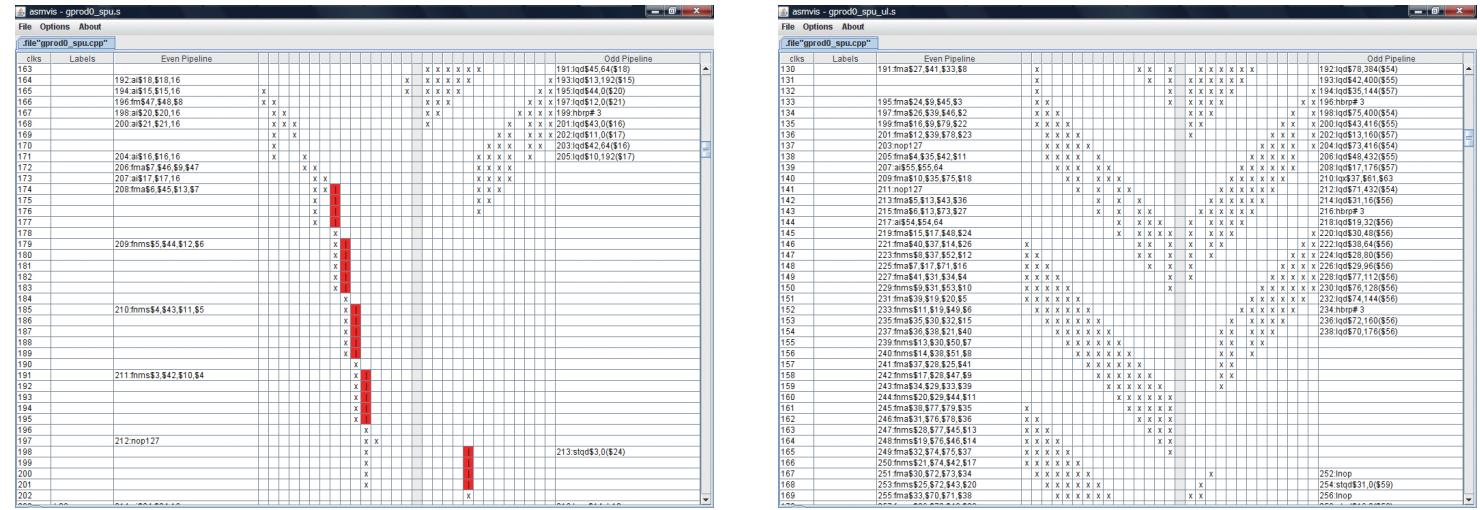

Figure 2: Dual-Pipeline optimization overview. (left is Normal calculations, and right is dual-issue optimizaions)

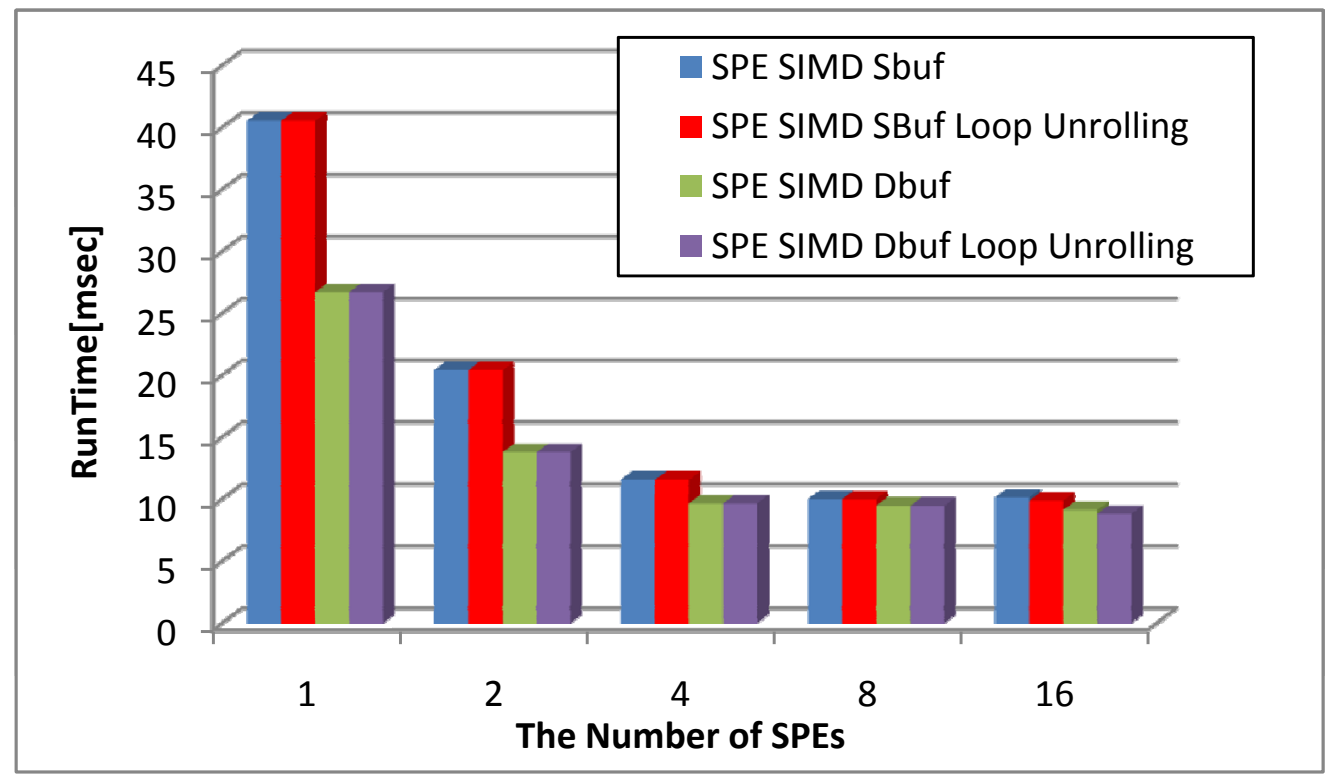

Figure 3: Result of Loop Unrolling Calculations

manual operation, and it widens the cord range where a compiler can optimize to use many registers. In addition, the dependency due to the register competition decreases, and one can conceal a stall.

Furthermore the total road/store number in the loop decreases, and can conceal the access latency to LS. These are important advantages. We should avoid the resister competition. For this purpose we must keep it in mind that the load from LS to register needs six cycles. It makes a high performance code to use no variable which is just used, or to rearrange the order of operations.

We show a calculation result of Loop Unrolling in Fig. 3. When we use 16 SPEs (SIMD and Double Buffering), it takes 8.8119 (msec), This is equivalent to 13 GFlops. Therefore, 
we achieve about 41 times speedup in comparison with the speed in the PPE simple substance (365.080039msec, 0.31GFlops) by tuning of the calculation technique in the SPE.

\section{Discussions}

In this report, we briefly described our trials to run the $S U(3)$ matrix multiplication code on Cell/B.E. at high performance. Combining several tuning techniques, we get over 20 GFLOPS(results of the latest our work), which is about one tenth of the theoretical peak speed.

This is probably much better than expected in the community. But we think more improvement is possible. There are several points worth to be considered:

1. Double buffering does not hide completely the transfer time. Multi (more than four) buffering seems to be necessary.

2. We waited the completion of the calculation, $c^{(i)}=a^{(i)} \times b^{(i)}$, and transfer back the result, $c^{(i)}$ to the main memory. This part has a room to be improved.

3. There may be more efficient data structure for $a, b$ and $c$ for the best use of SIMD.

In this experiment, we execute the calculation only once, and therefore we create a thread once. In real calculations, we must create threads many times, and it takes time to create a thread. Therefore, it is desirable to recycle the thread many times and to generate a thread once.

\section{Acknowledgement}

This work is supported by Grant-in-Aide for Scientific Research by Monbu-Kagaku-sho, Japan (20340055).

\section{References}

[1] F. Belletti et al., PoS (LATTICE 2007) 039.

[2] S. Motoki and A. Nakamura PoS (LATTICE2007) 040.

[3] G. Shi, V. Kindratenko and S. Gottlieb, PoS(LATTICE 2008) 026: Int. J. Parallel Prog. 37, 2009, pp488-507.

[4] H. Baier et al., PoS (LAT2009) 001.

[5] S. Williams, J. Shalf, L. Oliker, S. Kamil, P. Husbands and K. Yelick, Scientific Computing Kernels on the Cell Processor, International Journal of Parallel Programming, Vol.35, No.3, pp.263-298(2007).

[6] T. Kihara, H. Tadano, T. Sakurai, Implementation and performance evaluation of sparse matrix vector multiplication for mixed precision Krylov method on the Cell BE, IPSJ Transactions on Advanced Computing Systems, Vol. 1, pp. 51-60 (2008) (in Japanese).

[7] Khaled Z. Ibrahim, Francois Bodin, SIMDization and data management of the Lattice QCD computation on the Cell Broadband Engine, Scientific Programming, Vol.17, pp.153-172(2009). 